\title{
Sintese de evidências para políticas de saúde: enfrentamento da síflis congênita no âmbito da atenção primária à saúde
}

\author{
Evidence brief for health policy-making: facing congenital syphilis in primary health care
}

Mariana de Sales Dias', Emiliana Maria Gaiotto", Marcia Regina Cunha'", Lúcia Izumi Yasuko Nichiatalv

Resumo

Introdução: $O$ controle da sífilis congênita (SC) é um desafio para profissionais e gestores da Atenção Primária à Saúde (APS). Objetivo: Apresentar os resultados da síntese de evidências científicas pela qual foram identificadas opções para enfrentamento da SC que apoiam a APS. Método: Utilizou-se a ferramenta SUPPORT sugerida pela Rede para Políticas Informadas por Evidências (EVIPNet) para a busca de revisões sistemáticas e elaboração das opções para enfrentar o problema. As buscas foram realizadas em Biblioteca Virtual de Saúde, Cochrane Library, Embase, Health Evidence, Health Systems Evidence, Nice Evidence, Pubmed, Scopus. Resultados: Foram encontrados 310 estudos. Após seleção, analisaram-se 20 revisões sistemáticas que resultaram nas seguintes opções: 1) Ampliar a oferta de teste de triagem para todas as gestantes e parceiros sexuais; 2) Realizar tratamento com penicilina benzatina para gestantes e parceiros sexuais com teste de triagem positivo para sífilis; 3) Elaborar estratégias para notificar parceiros sexuais de pacientes com diagnóstico de infecção sexualmente transmissível (IST); 4) Realizar campanhas de saúde para informar sobre as medidas de prevenção da sífilis congênita; promover intervenções educacionais sobre o uso de preservativos, prevenção de IST e gravidez na adolescência. Conclusão: As evidências científicas apresentadas neste artigo têm potencial para apoiar gestores e profissionais da APS na construção de políticas de saúde para enfrentamento da SC.

Palavras-chave: Sífilis Congênita; Atenção Primária à Saúde; Política Informada por Evidências.

\footnotetext{
Mariana de Sales Dias (marianasalesdias@usp.br) é enfermeira, Mestre em Ciências pelo Programa de Mestrado Profissional em Enfermagem na Atenção Primária em Saúde no Sistema Único de Saúde da Escola de Enfermagem da Universidade de São Paulo (USP).

" Emiliana Maria Grando Gaiotto (emilianagaiotto@usp.br) é enfermeira, doutoranda no Programa de Pós-Graduação em Enfermagem da Escola de Enfermagem da Universidade de São Paulo.
}

Introduction: Control of congenital syphilis (CS) is a challenge for professionals and managers of Primary Health Care (PHC). Objective: To present the results of the synthesis of scientific evidences by which options for coping with CS were identified. Method: The SUPPORT tool suggested by the Evidence-Informed Policy Network (EVIPNet) was used to search for systematic reviews, with the identification of options to confront the problem. The searches were conducted in Biblioteca Virtual de Saúde, Cochrane Library, Embase, Health Evidence, Health Systems Evidence, Nice Evidence, Pubmed, Scopus. Results: There were 310 studies identified. After selection, we analyzed 20 systematic reviews that resulted in a set of options: 1) Expand the offer of screening test for all pregnant women and sexual partners; 2) To perform benzathine penicillin treatment for pregnant women and sexual partners with positive screening test for syphilis; 3) Develop strategies to notify sexual partners of patients with a diagnosis of sexually transmitted infections (IST); 4) Carry out health campaigns to inform the ways of preventing congenital syphilis; promote educational interventions on condom use, IST prevention, and teenage pregnancy. Conclusion: It is expected that the scientific evidence presented in this article may support managers and PHC professionals in the construction of health policies to confront CS.

Key words: Congenital Syphilis; Primary Health Care; Evidence-Informed Policy.
III Márcia Regina Cunha (marciareginacunha18@gmail.com) é enfermeira, Mestre em Ciências pelo Programa de Mestrado Profissional em Enfermagem na Atenção Primária em Saúde no Sistema Único de Saúde da Escola de Enfermagem Universidade de São Paulo e doutoranda no Programa de Pós-Graduação em Enfermagem da Escola de Enfermagem da Universidade de São Paulo.

Iv Lucia Yasuko Izumi Nichiatta (izumi@usp.br) é enfermeira, doutora, Professora Associada do Departamento de Enfermagem em Saúde Coletiva da Escola de Enfermagem da Universidade de São Paulo. 


\section{Enfrentamento da sífilis congênita na Atenção Primária à Saúde}

A sífilis congênita é uma doença causada pela bactéria Treponema pallidum, que é transmitida da gestante infectada para o feto, em razão de sua entrada por via transplacentária, embora também possa ocorrer durante a passagem do feto pelo canal do parto, se houver a presença de lesão ativa. ${ }^{1}$ Para triagem e diagnóstico da sífilis existem dois tipos principais de testes sorológicos: teste não treponêmico e teste treponêmico. Testes não treponêmicos são testes relativamente baratos, fáceis de realizar, sensíveis e quantitativos. Uma desvantagem dos testes não treponêmicos é que os falsos positivos podem ser causados por infecções virais agudas e por doenças autoimunes. 0 diagnóstico de sífilis deve ser confirmado em pessoas com teste positivo não treponêmico por meio de testes treponêmicos. ${ }^{2}$

Em 2011 o Ministério da Saúde permitiu que investigação da sífilis, especialmente em gestantes, comece com o uso de testes de triagem rápida para sífilis, que são testes treponêmicos cuja execução, leitura e interpretação dos resultados são feitas em no máximo 30 minutos. $^{3}$ Essa estratégia pode ser muito útil no apoio aos profissionais da APS para realizar captação e tratamento precoce de gestantes infectadas, a tempo de se prevenir a sífilis congênita. ${ }^{4}$

No Brasil, toda gestante deve ser testada para sífilis pelo menos três vezes: na primeira consulta de pré-natal (idealmente no primeiro trimestre da gestação), no terceiro trimestre (a partir da $28^{a}$ semana de gestação) e no momento do parto ou aborto, independentemente de exames anteriores. A parceria sexual, também deve ser testada para sífilis e outras Infecções Sexualmente Transmissíveis (IST). ${ }^{5}$

No âmbito do tratamento, conta-se com a penicilina benzatina, reconhecida pela $69^{\mathrm{a}}$ Assembleia Mundial da Saúde como um medicamento essencial para controle da transmissão vertical de sífilis, por atravessar a barreira transplacentária. As parcerias sexuais de gestantes com sífilis podem estar infectadas, mesmo apresentando testes imunológicos não reagentes; portanto, também devem ser tratadas. No caso das crianças expostas à sífilis durante o período gestacional, o tratamento deverá levar em consideração a situação clínica laboratorial da mãe. ${ }^{5}$

Embora a sífilis seja uma doença para a qual existem recursos diagnósticos e terapêuticos simples e de baixo custo, seu controle na gestação é uma tarefa complicada em decorrência do curto intervalo da gestação para a realização do seu diagnóstico e tratamento; pela dificuldade de abordagem de IST, principalmente durante a gestação; e provavelmente pelo desconhecimento da população e dos profissionais de saúde sobre a magnitude desse agravo e dos danos que ele pode causar. ${ }^{6}$

A Organização Mundial da Saúde lançou uma estratégia para eliminar a transmissão vertical da sífilis e do HIV que se baseia em quatro pilares: garantir um compromisso político sustentado; aumentar o acesso e a qualidade dos serviços de saúde materno-neonatal; monitorar e tratar as grávidas e os seus parceiros; estabelecer sistemas de vigilância, desenvolver indicadores e fortalecer os sistemas de monitoramento e avaliação. $^{7}$

Um importante estudo de abrangência nacional argumenta que um número baixo de casos de SC não indica necessariamente um bom programa de controle da transmissão vertical, uma vez que casos de SC podem estar ocorrendo, mas não estar sendo notificados. Já um número elevado de casos de SC possivelmente indica oportunidades perdidas de intervenção por falhas no processo de notificação, diagnóstico, tratamento adequado das gestantes e seus parceiros. ${ }^{8} \mathrm{Em}$ reunião realizada em 2018, tendo por base este 
citado estudo, especialistas e gestores que atuam na atenção primária à saúde em áreas de prevenção à IST da cidade de São Paulo, argumentaram que apesar dos esforços empreendidos e recomendações do Ministério da Saúde para triagem e tratamento serem realizadas nos serviços de atenção primária do município, a SC continua a aumentar expressivamente na cidade.

Para tornar realidade a erradicação da SC no Brasil, é necessário investir em capacitação profissional, sensibilização e padronização das condutas dos profissionais de saúde. Além disso, serviços de atenção primária à saúde devem passar por um processo de supervisão que ofereça apoio para a implementação das diretrizes recomendadas. $^{9}$

Diante dessas constatações, verificou-se que são inúmeros os desafios para reduzir a incidência dos casos de sífilis congênita. Nesse sentido, questiona-se quais são as evidências científicas que podem informar opções para o enfrentamento deste agravo.

\section{Objetivo}

Este artigo tem como objetivo apresentar os resultados de uma síntese de evidências científicas pela qual foram identificadas opções para o enfrentamento da sífilis congênita no âmbito da Atenção Primária à Saúde (APS).

\section{Métodos}

Para a elaboração da síntese de evidências, utilizou-se a ferramenta SUPPORT (Suporting policy relevant reviews and trials - Suporte a ensaios e revisões relevantes para as políticas) empregada pela Rede de Políticas Informadas por Evidências (EVIPNet). ${ }^{10}$ Essa ferramenta tem o objetivo de reduzir o abismo entre evidências científicas e as práticas nos serviços e nas políticas de saúde, por meio da tradução do conhecimento. ${ }^{11}$

A identificação de evidências que pudessem fornecer subsídios ao enfrentamento da sífilis congênita foi realizada por meio de uma seleção de revisões sistemáticas sobre intervenções efetivas para lidar com o problema. As revisões sistemáticas são vistas como uma das principais fontes de informação para a formulação de políticas. ${ }^{10}$

Foram acessadas as seguintes bases de dados: Biblioteca Virtual de Saúde, Cochrane Library, Embase, Health Evidence, Health Systems Evidence, Nice Evidence, Pubmed, Scopus. Os descritores utilizados foram "sífilis", "sífilis congênita", "transmissão vertical de doença infecciosa", "gestante", em português e inglês.

Após a definição das estratégias de busca, o processo de seleção foi realizado por duas pesquisadoras (MSD e EMG), de forma independente, em datas diferentes. Ambas identificaram os mesmos estudos. Inicialmente, foram excluídos estudos em duplicidade. Seguiu-se a leitura dos títulos e resumos para definição das revisões que seriam lidas na íntegra. A partir da leitura completa, as pesquisadoras entraram em consenso sobre quais estudos deveriam compor a síntese e realizaram o processo de extração de dados. Nessa fase foi elaborada uma planilha com o conjunto das informações detalhadas de cada estudo. A qualidade das revisões sistemáticas foi avaliada pelo instrumento AMSTAR - a measurement tool to assess the methodological quality of systematic reviews $^{12}$ e não foi utilizado um escore limite para exclusão dos estudos.

\section{Resultados}

De 310 estudos identificados, 20 foram incluídos nesta síntese. Na Figura 1 é apresentado o processo completo da seleção dos artigos. 
Figura 1 - Fluxo de informação com as diferentes fases da revisão sistemática.

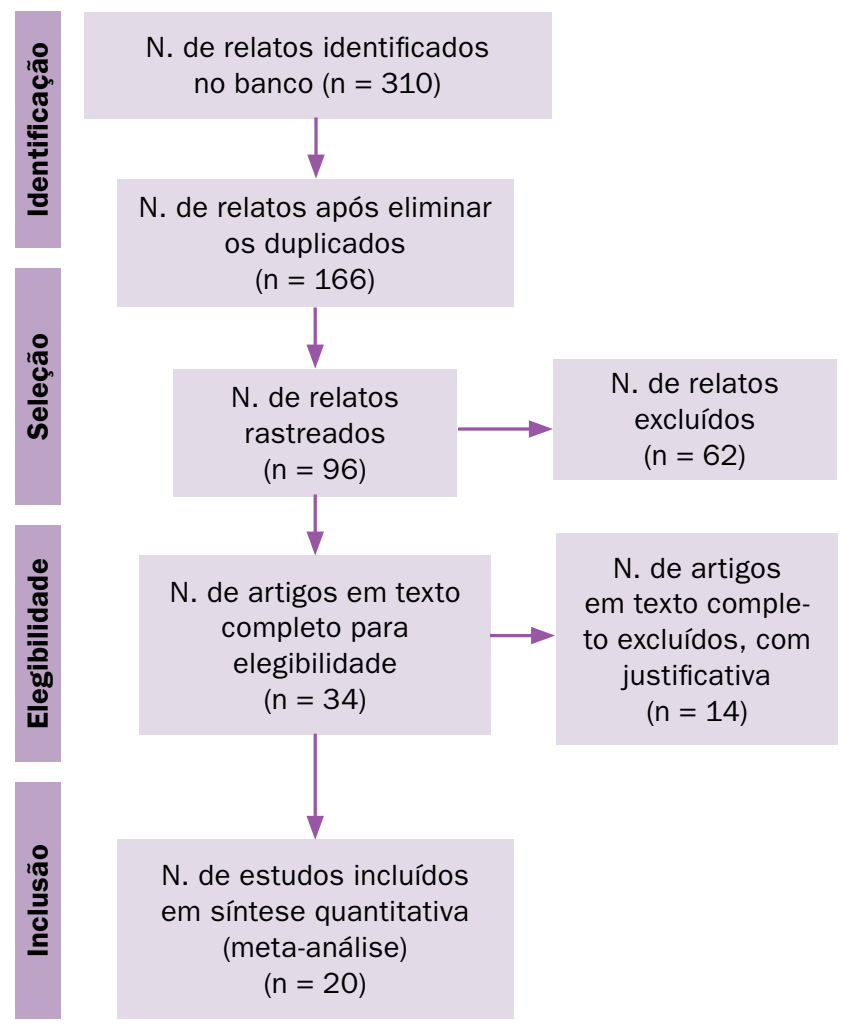

Vale destacar que nenhuma das revisões encontradas fez referência direta ao termo Atenção Primária à Saúde. Dessa forma, na elaboração sobre equidade e implementação das opções, foram agregadas considerações que tratam deste ponto de atenção. As opções de política não apresentam o mesmo nível de efetividade e exigem consideração sobre riscos e benefícios, bem como quanto a barreiras e facilitadores na implementação.

Essa síntese de evidências identificou quatro opções para a redução da SC, conforme apresentados a seguir.

\section{1) Ampliar a oferta de teste de triagem pa-} ra todas as gestantes.

Esta opção informa sobre a importância de oferecer testes rápidos de triagem dupla para sífilis e HIV, utilizando o mesmo cartucho, para todas as gestantes e parceiros, tanto na primeira consulta de pré-natal quanto no terceiro trimestre. Essa estratégia visa oportunizar tratamento imediato, prevenção de complicações decorrentes da transmissão vertical da doença e da coinfecção pelo HIV.

Os principais elementos que compõem essa opção são a realização de triagem para sífilis no local de atendimento do pré-natal, no primeiro e terceiro trimestres: ${ }^{13}$ implementação de teste rápido duplo para triagem de sífilis e $\mathrm{HIV}^{14,15} \mathrm{e}$ redução de morte fetal e natimortalidade, bem como a transmissão vertical de sífilis, por meio da triagem e tratamento da doença no início da gestação. ${ }^{16,17}$

A avaliação da sensibilidade e especifidade dos testes rápidos mostrou que, em configurações limitadas de recursos, o benefício da triagem usando testes rápidos supera em muito o risco de uma pequena proporção de casos perdidos em decorrência de resultados falso-negativos ou ao tratamento excessivo devido a uma infecção anterior. ${ }^{18}$

A maior parte dos estudos incluídos nas revisões sistemáticas que avaliaram a triagem pré-natal para reduzir a incidência de sífilis congênita foram realizados em países de baixa renda, média baixa renda, média alta renda. ${ }^{13-16,17,18,19}$

\section{2) Realizar tratamento com penicilina ben-} zatina para gestantes com teste de triagem positivo para sífilis.

Esta opção baseia-se na necessidade do tratamento das gestantes com diagnóstico de sífilis com penicilina benzatina, se possível no primeiro trimestre da gravidez. Além disso, o tratamento dos parceiros sexuais pode prevenir a reinfecção da gestante e a transmissão da sífilis congênita.

A penicilina é eficaz no tratamento da sífilis em gestantes em termos de curar a infecção na 
mulher e também prevenir a SC. A transmissão vertical da sífilis foi prevenida em 95\% a 98\% dos casos por meio do tratamento com penicilina. ${ }^{2}$ Complementando essa informação, outra revisão sistemática traz evidência de que pelo menos 2,4 milhões UI de penicilina benzatina aplicada até 28 dias antes do parto são eficazes no tratamento da sífilis em gestantes para prevenir a SC, com maior efeito quando administradas no início da gravidez (antes de 24 a 28 semanas). ${ }^{20}$

A sífilis não tratada contribui para o aumento de natimortos em diversas regiões do mundo. Em algumas áreas da África Subsaariana, cerca de $25 \%$ a $50 \%$ de todos os natimortos foram associados à sífilis. ${ }^{21}$ Outra revisão sistemática afirma que a natimortalidade na região das Américas associada à sífilis materna não tratada é um grave problema de saúde pública, que ocorreu numa proporção 6,8 vezes maior do que nas mulheres que não tiveram sífilis na gestação. ${ }^{22}$

\section{3) Elaborar estratégias para notificar IST e} tratar parceiros.

Esta opção trata de estratégias para notificar parceiros de pacientes com diagnóstico de IST, incluindo a sífilis, visando prevenir a reinfecção, disseminação e possíveis complicações da doença, como é o caso da SC.

Os principais elementos que compõem esta opção abrangem quatro estratégias principais para notificação do parceiro: a) Encaminhamento pelo paciente: o paciente diz a seus parceiros sexuais que eles precisam ser tratados; b) Terapia de parceiros acelerada: o paciente entrega medicação ou uma receita de medicação aos parceiros sem a necessidade de um exame médico do parceiro; c) Encaminhamento do provedor: o pessoal do serviço de saúde entra em contato com os parceiros e os notifica, após o consentimento do paciente; d) Encaminhamento por contrato: o paciente é encorajado a notificar os parceiros, mas o pessoal do serviço de saúde entrará em contato com eles se não visitarem o serviço de saúde até uma determinada data acordada com o paciente. ${ }^{23}$

As estratégias de notificação requerem que o paciente revele seu diagnóstico de IST aos parceiros e os encoraje a considerar o rastreamento ou o tratamento. Essa divulgação pode levar a benefícios como o tratamento bem-sucedido dos parceiros, o apoio emocional e a proteção da saúde de outras pessoas. Porém, também pode levar a estigma, rejeição, abuso físico e discriminação. ${ }^{23}$

\section{4) Promover ações educativas, por meio de campanhas de saúde com ênfase na sífilis congênita, intervenções relacionadas ao uso de preservativos, prevenção de IST e gravidez na adolescência.}

Esta opção aborda a promoção de campanhas de saúde na mídia para informar a população sobre as formas de prevenção da transmissão vertical de sífilis, com definição de indicadores em longo prazo; o incentivo ao uso de preservativos; a realização de intervenções escolares para prevenção de IST e gravidez na adolescência.

São considerados benefícios desta opção: a) modificações no estado de saúde de uma população por meio de campanhas de saúde de combate à sífilis; b) redução de IST em todo o mundo pela promoção do uso de preservativos; c) possibilidade de redução da gravidez na adolescência a partir de intervenções baseadas em incentivos que se concentrem em manter os jovens na escola secundária. ${ }^{24-26}$

Cada vez mais se percebe que os determinantes estruturais de saúde são questões importantes a serem abordados para melhorar os resultados sexuais e reprodutivos. ${ }^{24}$ 
Considerações sobre a equidade das opções e a sua implementação

A equidade é um dos princípios doutrinários do SUS que norteia as políticas de saúde. Seu sentido remete ao entendimento de que há necessidades de grupos específicos e que é possível realizar práticas que reconheçam as diferenças nas condições de vida e saúde e nas necessidades dos grupos sociais, considerando o direito à saúde. ${ }^{27}$ No caso das opções para o enfrentamento da SC, significa que as decisões sobre a implementação destas devem levar em consideração se essas opções poderão ou não influenciar negativamente as desigualdades entre os grupos sociais.

Nem todas as opções apresentadas têm necessariamente que ser implementadas de forma conjunta, uma vez que a aplicação prática deve considerar a viabilidade local, inserindo-se na governabilidade da tomada de decisão, independentemente da dimensão do sistema de saúde (nacional, regional ou local). Faz-se necessário considerar que a falta de infraestrutura, de recursos humanos, de definição do fluxo de trabalho no seguimento de pacientes com resultados reagentes a sífilis e aceitabilidade do teste podem ser barreiras à sua implementação na APS. ${ }^{4}$ Também é importante considerar as fragilidades e potencialidades da APS à implementação das opções, especialmente as localizadas no campo da cultura e representações sociais dos usuários e trabalhadores de saúde.

A organização do processo de assistência no pré-natal compromete a efetividade do tratamento da sífilis durante a gestação, o que torna evidente a urgência de se repensar as práticas relacionadas às oportunidades de tratamento da sífilis, conferindo maior responsabilidade dos diversos pontos da rede, especialmente nas unidades de atenção primária à saúde. ${ }^{28}$

\section{Considerações Finais}

Quatro opções foram identificadas para o enfrentamento da SC no âmbito da APS a partir de vinte revisões sistemáticas selecionadas. Porém nenhuma das revisões sistemáticas encontradas para compor esse estudo fez referência direta ao termo Atenção Primária a Saúde, o que pode denotar uma lacuna de evidências para a abordagem da SC nesse ponto de atenção.

Existem políticas públicas para a erradicação da SC no Brasil, nas quais as opções propostas nesse estudo também são abordadas. Porém, a maioria dos serviços de saúde apresenta fragilidades na implementação das diretrizes recomendadas. Assim, reconhece-se a relevância dessa síntese de evidências científicas em reafirmar as recomendações para enfrentar a SC.

Um limite encontrado nesse estudo foi a ausência de revisões sistemáticas que tratem do universo das relações sociais/familiares no contexto da sexualidade. Há diversos estudos que abordam a questão clínica da sífilis, com enfoque em diagnóstico e terapêutica. No entanto, sugere-se a produção de revisões sistemáticas que extrapolem o lado biológico da doença e analisem fatores referentes ao contexto de vulnerabilidade dos indivíduos portadores de IST.

Espera-se que as evidências científicas apresentadas nesse estudo possam apoiar gestores e profissionais da APS no avanço da construção de políticas de saúde para enfrentamento da SC, e que futuramente um diálogo deliberativo possa subsidiar a implementação de algumas das opções abordadas na síntese.

Conflitos de interesse: Os autores declaram não haver conflitos de interesse que possam ter influenciado na realização estudo.

\section{Parecer do Comitê de Ética em Pesquisa:} 88268018.6.3001.0086 


\section{Referências}

1. Berman SM. Maternal syphilis: Pathophysiology and treatment. Bull World Health Organ. 2004; 82(6):433-38.

2. Walker GJA. Antibiotics for syphilis diagnosed during pregnancy. Cochrane Database of Systematic Reviews; 2001.

3. Ministério da Saúde (BR). Portaria $n^{\circ} 3.242$, de 30 de Dezembro de 2011. Dispõe sobre o Fluxograma Laboratorial da Sífilis e a utilização de testes rápidos para triagem da sífilis. Brasília; 2011.

4. Nascimento DSF, Silva RC, Tártari DO, Cardoso EK. Relato da dificuldade na implementação de teste rápido para detecção de sífilis em gestantes na Atenção Básica do SUS em um município do Sul do Brasil. Rev Bras Med Fam Comunidade. 2018;13(40):1-8.

5. Ministério da Saúde (BR). Protocolo Clínico e Diretrizes Terapêuticas para Prevenção da Transmissão Vertical de HIV, Sífilis e Hepatites Virais. Brasília (DF); 2018.

6. Domingues RMSM, Saraceni V, Hartz ZMDA, Leal MDC. Sífilis congênita: evento sentinela da qualidade da assistência pré-natal. Rev Nutr. 2013; 47(1):147-57.

7. World Health Organization. The Global elimination of congenital syphilis: rationale and strategy for action; 2007.

8. Domingues RMSM, Leal MC. Incidência de sífilis congênita e fatores associados à transmissão vertical da sífilis: dados do estudo Nascer no Brasil; 2016.

9. Rocha AFB, Araújo MAL, Miranda AE, Leon RGP, Junior GBS, Vasconcelos LDPG. Management of sexual partners of pregnant women with syphilis in northeastern Brazil: a qualitative study. BMC Health Services Research; 2019.

10. Lavis JN, Oxman AD, Lewin S, Fretheim A. SUPPORT tooll for evidence-informed health policymaking (STP). Introduction. Health Research Policy and Systems; 2009.

11. Vettore M. O abismo entre o saber e o fazer: a experiência do município de Piripiri com o Núcleo de Evidências em Saúde no planejamento de política em saúde; 2011.

12. Shea BJ, Grimshaw JM, Wells GA, Boers M, Andersson N, Hamel C, et al. Development of AMSTAR: a measurement tool to assess the methodological quality of systematic reviews. BMC Medical Research Methodology. 2007; 7(10).

13. Shahrook S, Mori R, Ochirbat T, Gomi H. Strategies of testing for syphilis during pregnancy; 2014.

14. Harriet DG, Peeling RW, Kamb ML, Toskin I, Wi TE, Taylor MM. A systematic review and meta-analysis of studies evaluating the performance and operational characteristics of dual point-of-care tests for HIV and syphilis; 2016.

15. Swartzendruber A, Steiner RJ, Adler MR, Kamb ML, Newman LM. Introduction of rapid syphilis testing in antenatal care: A systematic review of the impact on HIV and syphilis testing uptake and coverage; 2015.

16. Di Mario S, Say L, Lincetto O. Risk factors for stillbirth in developing countries: a systematic review of the literature; 2007.

17. Hawkes S, Matin N, Broutet N, Low N. Effectiveness of interventions to improve screening for syphilis in pregnancy: a systematic review and meta-analysis; 2011.

18. Jafari Y, Peeling RW, Shivkumar S, Claessens C, Joseph L, Pai NP. Are Treponema pallidum Specific Rapid and Point-of-Care Tests for Syphilis Accurate Enough for Screening in Resource Limited Settings? Evidence from a Meta-Analysis; 2013.

19. Rogozinska E, Kara-Newton L, Zamora JR, Khan KS. On-site test to detect syphilis in pregnancy: a systematic review of test accuracy studies; 2017.

20. Blencowe H, Cousens S, Kamb M, Berman S, Lawn JE. Lives Saved Tool supplement detection and treatment of syphilis in pregnancy to reduce syphilis related stillbirths and neonatal mortality. BMC Public Health; 2011.

21. Bhutta et al. Effectiveness of interventions to screen and manage infections during pregnancy on reducing stillbirths: a review. BMC Public Health; 2011.

22. Lauren A, Serruya S, Duran P. Gestational syphilis and stillbirth in the Americas: a systematic review and meta-analysis; 2015.

23. Ferreira A, Young T, Mathews C, Zunza M, Low N. Strategies for partner notification for sexually transmitted infections, including HIV; 2013.

24. Mason-Jones AJ, Sinclair D, Mathews C, Kagee A, Hillman A, Lombard C. School-based interventions for preventing HIV, sexually transmitted infections, and pregnancy in adolescents; 2014.

25. Moreno R, Nababan HY, Ota E, Wariki WMV, Ezoe S, Gilmour S, Shibuya K. Structural and community-level interventions for increasing condom use to prevent the transmission of HIV and other sexually transmitted infections; 2014.

26. Saraceni V, Leal M do C, Hartz ZM de A. Avaliação de campanhas de saúde com ênfase na sífilis congênita: uma revisão sistemática. Rev Bras Saúde Matern Infant. 2005; 5(3):263-73.

27. Barros FPC, Sousa MF. Equidade: seus conceitos, significações e implicações para o SUS São Paulo: Saúde Soc. 2016; 25(1):9-18.

28. Sanine et al. Sífilis Congênita: avaliação em serviços de Atenção Primária do estado de São Paulo, Brasil. BIS, Bol. Inst. Saúde. 2016; 17(2):1809-7529. 